\title{
Traumatismes de L'Abdomen et du Périnée par Encornements de Boufs au Centre Hospitalier Régional de Dapaong, Togo
}

\author{
Biréga Koutora, \\ Abossisso Sakiye, \\ Yves Aziz R. Nacanabo, \\ Séna A. Tchalla, \\ Efoé-Ga Amouzou, \\ Mahèza Akpanahe, \\ Tarek Alassani,
} Département de Chirurgie et Spécialités Chirurgicales, Faculté des Sciences de la Santé, Université de Lomé, Togo

Kokou K. Kanassoua, Badjona Songne-Gnamkoulamba,

Service des Urgences Chirurgicales, CHU Sylvanus Olympio, Togo

Faculté des Sciences de la Santé, Université de Lomé, Togo

Doi:10.19044/esj.2019.v15n24p178 URL:http://dx.doi.org/10.19044/esj.2019.v15n24p178

Résumé

Objectifs: Décrire les aspects épidémiologiques, lésionnels et thérapeutiques des traumatismes de l'abdomen et du périnée par encornements de bœufs au Centre Hospitalier Régional de Dapaong. Méthodologie : Etude rétrospective descriptive incluant les dossiers des blessés de l'abdomen et du périnée par encornements de bœufs, pris en charge dans le service de chirurgie du CHR Dapaong, de Janvier 2016 à Décembre 2018 (3 ans). Résultats : Trente-quatre patients ont été pris en charge pour lésions traumatiques de l'abdomen et du périnée par coups de cornes de bœufs. Il s'agissait de 31 hommes et 3 femmes d'âge moyen de 18,47 $\pm 16,14$ ans (extrêmes : 6 ans et 70 ans). La majorité des victimes avait moins de 15 ans, et était des gardes troupeaux. Le bilan lésionnel au niveau abdominal notait 9 cas de contusion et 22 cas de plaies dont 19 cas de plaies pénétrantes. Les lésions viscérales étaient variées et souvent associées. Au niveau périnéal, on notait 3 cas de plaies dont un cas de lésion rectale associée. La prise en charge était chirurgicale dans la majorité des cas. L'évolution à court terme était marquée par une infection locale dans 8 cas $(23,5 \%)$. Le pronostic était bon dans tous 
les cas. Conclusion : Les encornements de bœufs constituent une cause fréquente de traumatismes de l'abdomen et du périnée au CHR Dapaong. L'évolution après prise en charge adéquate est souvent favorable.

Mots-clés: Abdomen, Encornement, Périnée, Togo

\title{
Abdomen and Perineum Bull Horn Trauma at Regional Hospital of Dapaong, Togo
}

\author{
Biréga Koutora, \\ Abossisso Sakiye, \\ Yves Aziz R. Nacanabo, \\ Séna A. Tchalla, \\ Efoé-Ga Amouzou, \\ Mahèza Akpanahe, \\ Tarek Alassani,
} Département de Chirurgie et Spécialités Chirurgicales, Faculté des Sciences de la Santé, Université de Lomé, Togo

Kokou K. Kanassoua, Badjona Songne-Gnamkoulamba, Service des Urgences Chirurgicales, CHU Sylvanus Olympio, Togo Faculté des Sciences de la Santé, Université de Lomé, Togo

\begin{abstract}
Background: This paper focuses on describing the epidemilogical, lesional, and therapeutic aspects of abdomen and perineum bull horn trauma at Regional Hospital of Dapaong. Methodology: It is a descriptive retrospective study, which includes patients treated for abdomen and perineum bullhorn trauma, in the surgery department of Regional Hospital of Dapaong from January 2016 to December 2018 (3 years). Results: 34 patients were treated for abdominal and perineal bullhorn injuries. They comprised of 31 men and 3 women, with an average age of $18.47 \pm 16.14$ years old (extremes: 6 and 70 years old). They were mainly less than 15 years old and were herd guards. The lesion report noted 9 cases of abdominal blunt trauma, 22 cases of abdominal wounds including 19 cases of penetrating wounds, and 3 cases of perineal wounds including 1 case of associated rectal lesion. Visceral lesions were varied and often associated. The care was mainly surgical. The
\end{abstract}


short term evolution was marked by a local infection in 8 cases $(23.5 \%)$. The prognosis was good in all cases. Conclusion: Bullhorn trauma is a frequent cause of abdomen and perineum injuries at Regional Hospital of Dapaong. The outcome after adequate care is often favourable.

Keywords: Abdomen, Bullhorn, Perineum, Togo

\section{Introduction}

Les lésions traumatiques par coups de cornes constituent une entité particulière par leur mécanisme lésionnel et leurs types qui sont différents de la traumatologie civile (Mokni, 2010 ; Chambres, 2005). Ces lésions sont fréquentes dans les régions à tradition taurine, où le taureau est impliqué dans des activités sportives et festives, et dans d'autres spectacles. Il s'agit : de l'Espagne, du Portugal, du Mexique, du sud de la France, de certains Etats d'Amérique latine ; et en Afrique à Madagascar avec la pratique de la corrida malgache (Randrianambinima, 2018; Mokni, 2010; Rudloff, 2006). Toutefois, ces lésions traumatiques par encornements peuvent être rencontrées dans des circonstances autres que les spectacles publics. Ainsi, en milieu rural où les populations vivent souvent essentiellement de l'élevage et de l'agriculture, et où le bœuf est utilisé pour des activités domestiques et des travaux champêtres, les traumatismes par encornements accidentels de bœufs y sont souvent rencontrés (Basbug, 2016 ; Gajbhiye, 2016 ; Santhosh, 2013). La topographie lésionnelle est variable selon les circonstances. Si au cours des courses taurines les lésions du membre pelvien en particulier de la cuisse sont prédominantes en raison de son exposition aux cornes pendant le spectacle, dans les accidents domestiques et champêtres, c'est souvent l'abdomen et le périnée qui sont les sièges des lésions (Mokni, 2010 ; Santhosh, 2013). A travers cette étude, nous rapportons l'expérience du service de chirurgie du Centre Hospitalier Régional (CHR) de Dapaong dans le nord - Togo sur les traumatismes de l'abdomen et du périnée par encornements de bœufs dans leurs aspects épidémiologiques, lésionnels et thérapeutiques.

\section{Méthodologie}

Il s'est agi d'une étude rétrospective descriptive couvrant la période de Janvier 2016 à Décembre 2018 (3 ans), incluant les dossiers des blessés de l'abdomen et du périnée par coups de cornes de bœufs, pris en charge dans le service de chirurgie du CHR de Dapaong. Les traumatismes abdominaux et périnéaux survenus dans d'autres circonstances n'ont pas été inclus dans l'étude, de même que les encornements d'autres régions anatomiques du corps. Les données ont été collectées à partir des registres d'admission des urgences et réanimation chirurgicale, d'anesthésie-réanimation du bloc opératoire, de comptes rendus opératoires ; et des dossiers rédigés des patients. 
Tous les patients avaient été admis à la salle des urgences et réanimation chirurgicale. Ils avaient bénéficié : d'un traitement médical associant des antalgiques et d'autres médications selon le besoin; des antibiotiques, d'une séro-anatoxinothérapie antitétanique et d'un parage de la plaie du point d'impact lorsqu'il s'agissait d'une plaie ; et d'un traitement spécifique suivant les lésions présentées.

Les paramètres étudiés étaient : les paramètres sociodémographiques (âge, sexe, profession), les aspects lésionnels (circonstances de survenue, siège et type de lésions), les aspects thérapeutiques et l'évolution.

Le traitement des données a été fait à partir du tableur Excel 2013. Les images ont été traitées sur le logiciel Paint.

\section{Résultats}

\section{Aspects épidémiologiques}

Durant la période d'étude, 82 patients ont été admis dans le service de chirurgie pour des traumatismes de l'abdomen ou du périnée toutes circonstances confondues, dont $34(41,4 \%)$ par encornements de bœufs. Les traumatismes de l'abdomen et du périnée par encornements de bœufs représentaient $91,9 \%$ de l'ensemble des lésions traumatiques par encornements de bœufs. La répartition des patients victimes d'encornements de bœufs selon le siège des lésions est résumée dans le Tableau I. L'âge moyen des patients étaient de 18,4 $\pm 16,1$ ans (extrêmes : 6 ans et 70 ans) avec une prédominance masculine (sex-ratio $=10,3)$. La tranche d'âge de 6 à 15 ans était la plus représentée (24 cas / 70,6\%). Selon la profession, les gardes troupeaux étaient majoritaires (16 cas / 47,1\%). Les paramètres épidémiologiques sont récapitulés dans le Tableau II.

\section{Aspects lésionnels}

Tous les patients avaient été accidentellement agressés par un coup de cornes de bœuf. Les lésions siégeaient à l'abdomen dans 31 cas $(91,1 \%)$ et au périnée dans 3 cas $(8,9 \%)$. Les lésions abdominales étaient représentées par les contusions abdominales dans 9 cas $(26,4 \%)$ et les plaies dans 22 cas $(64,7 \%)$ dont 19 cas $(55,8 \%)$ de plaies pénétrantes. Le caractère pénétrant de la plaie abdominale avait été évoqué sur la base d'une éviscération dans tous les 19 cas. La figure 1 est un exemple d'éviscération chez un patient de 16 ans victime d'un encornement de bœuf. Le caractère non pénétrant de la plaie était conclu après son exploration sous anesthésie locale. Les lésions du périnée étaient des plaies dans les trois cas, dont un cas de lésion rectale associée.

Selon le type de viscères lésés, l'intestin grêle était le plus atteint (21 cas / 61,7\%) suivi de la rate (11 cas / 32,3\%). Le Tableau III montre la répartition des patients selon le type de traumatisme et le type de viscères lésés. Les lésions viscérales étaient associées dans certains cas. Ainsi avait-on 
noté : une perforation de l'estomac associée à une perforation du côlon transverse dans 2 cas, une plaie du foie associée à une perforation du grêle dans un cas, une rupture de la rate associée à des plaies du grêle et à une contusion du mésentère dans 2 cas, une plaie du mésentère et du grêle dans 2 cas, et une fissure de la rate associée à un hématome retro-péritonéal dans un cas.

\section{Aspects thérapeutiques}

Une laparotomie avait été faite dans tous les cas de plaie pénétrante et dans 5 cas de contusion abdominale. Le traitement des lésions intraabdominales était fonction des lésions observées. Le Tableau IV récapitule les différents gestes opératoires effectués selon les lésions. Les cas de plaie non pénétrante de l'abdomen étaient traités par un parage et une suture de la plaie avec une surveillance en hospitalisation. Les plaies du périnée étaient traitées par un parage et des bains de siège. Une colostomie latérale iliaque gauche avait été réalisée dans un cas de plaie périnéale avec lésion rectale associée. Le rétablissement de la continuité avait été fait dans un délai de 2 mois. Un traitement médical et une surveillance avaient été institués dans 4 cas de contusion abdominale.

\section{Aspects évolutifs}

L'évolution à court terme était marquée par une infection locale dans 8 cas $(23,5 \%)$. Cette infection avait bien évoluée sous les soins locaux et traitement médical. La durée moyenne d'hospitalisation était de $4,4 \pm 2,1$ jours (extrêmes : 1 jour et 12 jours). Deux patients avaient présenté une éventration après un recul respectivement de 6 mois et 13 mois. Aucun cas de décès n'avait été enregistré.

\section{Discussion}

Les encornements de bœufs constituent une cause fréquente de traumatismes de l'abdomen et du périnée au CHR Dapaong. Ils y représentent 41,4\% des étiologies des traumatismes abdomino-périnéaux. Cette proportion est supérieure à celle rapportée dans d'autres études. En effet, Maske et collaborateurs (Maske, 2016) en Inde rapportaient une proportion de $8 \%$. Pour Sambo et collaborateurs (Sambo, 2016) au Benin, les agressions de bœufs représentaient $27,5 \%$ des causes de traumatismes abdominaux. Cette différence pourrait s'expliquer par le fait que cette étude a été réalisée dans une région où l'agriculture et l'élevage des bœufs occupent une place importante dans la vie des populations. Le bœuf est élevé non seulement pour son rôle dans l'alimentation, mais aussi pour son utilité dans les activités domestiques notamment la traction des charrettes pour le transport des marchandises, et dans les travaux champêtres avec la culture attelée. 
L'implication du bœuf dans ces activités augmente le risque d'encornement pour les personnes en contact. En effet, dans cette série, la majorité des patients étaient des personnes en contact avec les bœufs (gardes troupeaux, agriculteurs). Selon les études, la majorité des victimes d'encornements sont soit les propriétaires des bœufs, soit des travailleurs responsables de leur surveillance (Dogan, 2008). Le bœuf est normalement un animal calme, mais un comportement agressif peut être affiché sans raison apparente (Oster, 1994).

Les lésions traumatiques par encornements peuvent siéger dans n'importe quelle partie du corps. Cependant, la topographie lésionnelle est variable suivant les circonstances concourant à ces traumatismes. Dans les spectacles publics comme les corridas, le membre pelvien en particulier la cuisse constitue la localisation première des encornements (61\%) en raison de son exposition aux cornes du taureau pendant le spectacle (Randrianambinima, 2018 ; Mokni, 2010). Des travaux rapportant des lésons traumatiques par encornements de bœufs dans des circonstances d'agression accidentelle font état de la fréquence des lésions abdomino-périnéales et des organes génitaux externes (Singh, 2015; Santhosh, 2013; Rabenjamina, 2000). Le siège des lésions varie en fonction de la taille de l'animal et de sa position au moment de l'attaque, mais aussi de la taille de la victime (Randrianambinima, 2018). Les lésions engendrées, de gravité variable, vont de simples contusions aux plaies, et dépendent non seulement de la partie de la corne blessante (plat de corne ou pointe de la corne), mais aussi de la force d'attaque de l'animal. Ainsi, au niveau abdominal, une blessure par le plat de corne va provoquer une contusion abdominale et une blessure par la pointe de la corne va provoquer une plaie. La profondeur de la plaie et la gravité des lésions intra-abdominales sont fonction de la force de pénétration, du poids et de la vitesse de l'animal au contact (Mokni, 2010). Les plaies par encornements possèdent des caractéristiques qui les rendent différentes des plaies par blessure civile. Elles sont en effet caractérisées par des orifices d'entrée et parfois de sortie, des trajets, des cavités, des rebondissements et culs-de-sac, avec un très grand risque infectieux (Gajbhiye, 2016 ; Mokni, 2010).

Le caractère contaminé des plaies par encornements justifie le parage minutieux des plaies aux points d'impact, l'usage des antibiotiques et la séroanatoxinothérapie antitétanique. Les blessures par cornes sont en effet des blessures sales dont l'incidence d'infection est de l'ordre de 22 à $42 \%$ (Chambres, 2005). Dans cette étude, cette incidence était de 23,5\%. Le traitement spécifique est fonction des lésions. Les contusions abdominales de même que les plaies non pénétrantes par encornements nécessitent une surveillance car des lésions intra-abdominales sont toujours possibles. 


\section{Conclusion}

Les lésions traumatiques abdomino-périnéales par encornements de bœufs sont fréquentes au CHR Dapaong. Elles sont dominées au niveau abdominal par les plaies pénétrantes avec des lésions viscérales souvent multiples. Les plaies périnéales peuvent être associées à des lésions rectales. L'évolution de ces lésions après une prise en charge adéquate est le plus souvent favorable.

Conflit d'intérêt : aucun

Remerciements: Nos remerciements à tout le personnel du service de chirurgie du CHR Dapaong. Mention spéciale au chef de service et aux surveillants des différents pavillons pour avoir facilité la collecte des données.

\section{Annexe}

Tableau I. Répartition des patients victimes d'encornements selon le siège des lésions

\begin{tabular}{lrr}
\hline & Effectif & $\%$ \\
\hline Abdomen & 31 & 83,8 \\
Périnée & 3 & 8,1 \\
Organes génitaux externes & 1 & 2,7 \\
Cou & 1 & 2,7 \\
Epaule & 1 & 2,7 \\
Total & 37 & 100 \\
\hline
\end{tabular}

Tableau II. Récapitulatif des paramètres épidémiologiques

\begin{tabular}{ccrr}
\hline & & Effectif & $\%$ \\
\hline Sexe & Masculin & 31 & 91,2 \\
& Féminin & 3 & 8,8 \\
Tranche d'âge & {$[6-15[$} & 24 & 70,6 \\
& {$[15-30[$} & 2 & 5,9 \\
& {$[30-45[$} & 5 & 14,7 \\
Profession & $145-60[$ & 1 & 2,9 \\
& $\geq 60$ & 2 & 5,9 \\
& & & \\
& Gardes troupeaux & 16 & 47,1 \\
& Agriculteurs / Eleveurs & 7 & 20,6 \\
& Elèves & 4 & 11,7 \\
& Revendeurs & 3 & 8,8 \\
& Autres* & 4 & 11,8 \\
\hline
\end{tabular}

Autres* $=$ enfants non scolarisés ni gardes troupeaux, adultes sans emploi. 
Tableau III. Répartition des organes atteints en fonction du type de traumatisme.

\begin{tabular}{lrrrr}
\hline & $\begin{array}{r}\text { Plaie de } \\
\text { l'abdomen } \\
\mathrm{n}(\%)\end{array}$ & $\begin{array}{r}\text { Contusion } \\
\text { abdominale } \\
\mathrm{n}(\%)\end{array}$ & $\begin{array}{r}\text { Plaie du } \\
\text { périnée } \\
\mathrm{n}(\%)\end{array}$ & $\begin{array}{r}\text { Total } \\
\mathrm{n}(\%)\end{array}$ \\
\hline Estomac & $2(5,9)$ & 0 & 0 & $2(5,89)$ \\
Grêle & $19(55,9)$ & $2(5,9)$ & 0 & $21(61,8)$ \\
Côlon & $2(5,9)$ & 0 & 0 & $2(5,9)$ \\
Rectum & 0 & 0 & $1(2,9)$ & $1(2,9)$ \\
Rate & $4(11,7)$ & $7(20,6)$ & 0 & $11(32,3)$ \\
Foie & 0 & $1(2,9)$ & 0 & $1(2,9)$ \\
Mésentère & $3(8,8)$ & $1(2,9)$ & 0 & $4(11,7)$ \\
Retropéritoine & 0 & $1(2,9)$ & 0 & $1(2,9)$ \\
\hline
\end{tabular}

Tableau IV. Répartition des gestes chirurgicaux réalisés en fonction des lésions viscérales

\begin{tabular}{lccc}
\hline & Effectif & Gestes réalisés & Effectif \\
\hline Perforation de l'estomac & 2 & Excision - suture & 2 \\
Perforations du grêle & 21 & Excision - suture & 13 \\
& & Résection - anastomose & 8 \\
Perforation du côlon & 2 & Excision - suture & 2 \\
Plaie du rectum & 1 & Excision - suture & 1 \\
Rupture de la rate & 6 & Splénectomie totale & 6 \\
Fissure de la rate & 5 & Traitement conservateur & 3 \\
& & Splénectomie totale & 2 \\
Plaie du foie & 1 & Packing & 1 \\
Plaie du mésentère & 2 & Suture & 2 \\
Hématome rétro- & & Respect & 1 \\
péritonéal & 1 &
\end{tabular}

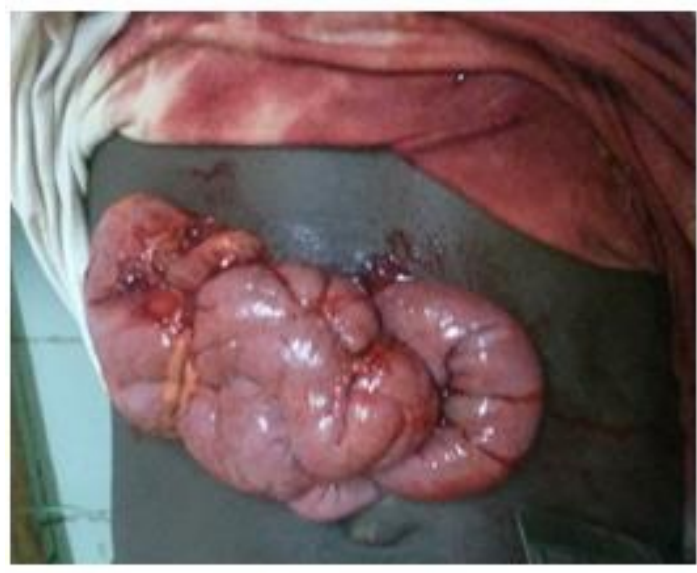

Crânial

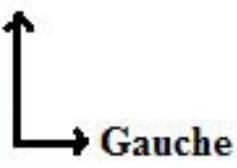

Figure 1. Eviscération par encornement de bøuf chez un patient de 16 ans 


\section{References:}

1. Basbug, HS., Gocer, H., Gunerhan, Y., \& Oizisik, K. (2016). An unusual case of isolated femoral vein injury after bull gore. AMAJ; 3 : 75-7.

2. Chambres, O., Thaveau, F., Gabbai, M., Giraud, C., Gouffrant, JM., \& Kretz, JG. (2005). Une discipline atypique : La chirurgie taurine. A propos de 2 observations. Ann Chir; $130: 340-5$.

3. Dogan, KH., Sunam, GS., Erkol, Z., Serafettin, D., Zerrin, E., Guven, SS., et al. (2008). Injuries and deaths occuring as a result of bull attack. J of Agromedicine; 13(3) : 191-6.

4. Gajbhiye, AS., Shamkuwar, A., Bokade, A., Nasare, V., Jehughale, K., \& Agrawal, A. (2016). Surgical management of bull horn injury. Int Surg J; 3(4) : 2041-5.

5. Maske, AN. \& Deshmukh, SN. (2016). Traumatic abdominal injuries : our experience at rural tertiary care center. Int Surg J; 3(2) : 543-8.

6. Mokni, T. (2010). Accidents liés aux corridas. Urgences; 42 : 457-67.

7. Osta, R. \& Rodellar, C. (1994). Effect of genetic factors on the criteria of stress in fighting bulls. Vet Res; 25(5) : 442-9.

8. Rabenjamina, FR. \& Rakotozafy, G. (2000). Une plaie abdominodiaphragmatique droite par encornement de zébu. Ann Chir; 125 : 497.

9. Randrianambinima, F., Jonatana, AD., Randrianambinima, H., Razafimanjato, NNM., Rakotoarisao, AJC., \& Rakotovao, HJL. (2018). Les traumatismes thoraciques par encornement de zebu à Antananarivo Madagascar. J Func Vent Pulm; 26(9) : 14-8.

10. Rudloff, U., Gonzalez, V., Fernandez, E., Holguin, E., Rubio, G., Lomelin, J., et al. (2006). Chirurgica Taurina : a 10-year experience of bullfight injuries. J of Trauma and Acute Care Surg; 61(4) : 970-4.

11. Sambo, BT., Hodonou, AM., Allode, AS., Mensah, E., Youssouf, M., \& Mehinto, D. (2016). Aspects épidémiologiques, diagnostiques et thérapeutiques des traumatismes abdominaux à Bembéréké-Nord Bénin. Eur Sci J; 12(9) : 395-405.

12. Santhosh, R., Barad, AK., Ghalige, HS., Sridartha, K., \& Sharma, B. (2013). Perineal bull gore with urinary bladder perforation and pneumoperitoneum. J of Clinical and Diagnostic Res; 7(5) : 902-4.

13. Singh, B., Kumar, A., Kaur, A., \& Singla, RL. (2015). Bullhorn hernia : a rare traumatic abdominal wall hernia. Niger J Surg; 21(1) : 63-5. 